\title{
INVESTIGACIÓN/RESEARCH
}

\section{RTVE SIN PUBLICIDAD: UN MODELO DE FINANCIACIÓN EN ESTADO DE EMERGENCIA}

\begin{abstract}
Dimitrina Jivkova-Semova ${ }^{1}$ : Universidad Complutense de Madrid. España d.jivkova@ccinf.ucm.es
\end{abstract}

\section{RESUMEN}

En una comunicación de 30 de septiembre de 2010 la Comisión Europea declaró su decisión de exigir a Francia y España que supriman las tasas específicas sobre el volumen de negocios de los operadores de telecomunicaciones. La Comisión considera que las tasas impuestas por ambos países al efecto de compensar la pérdida de ingresos en concepto de publicidad en los canales de televisión públicos son incompatibles con las normas de la UE en materia de telecomunicaciones. Este acto crea muchas incógnitas ante el actual modelo de financiación de la Corporación RTVE. En este artículo, intentamos analizar la situación económica- financiera de la radiotelevisión pública en España buscando al mismo tiempo ejemplos de gestión de países como Francia, Reino Unido y Alemania

PALABRAS CLAVE: Radiotelevisión pública- Financiación- Legislación europeaSector audiovisual y de telecomunicaciones

\section{RTVE WITHOUT ADVERTISING: A FUNDING MODEL IN STATE OF EMERGENCY}

\begin{abstract}
In its communication of 30 September, 2010 the European Commission said its decision to require France and Spain to abolish the specific rates on turnover of telecommunications operators. The Commission considers that the taxes imposed by both countries in order to compensate the loss of revenue from advertising on public television are incompatible with EU standards in telecommunications. This act

\footnotetext{
${ }^{1}$ Autor correspondiente

Dimitrina Jivkova-Semova: Profesora de la Universidad Complutense de Madrid, España.

Correo: $\underline{\text { d.jivkova@ccinf.ucm.es }}$
} 
creates a lot of unknowns in the current funding model of the RTVE Corporation. In this article, we attempt to analyze the economic and financial situation of the public broadcasting in Spain considering management examples from other countries: France, Britain and Germany.

KEY WORDS: Funding Model, European Legislation, Private Broadcasting, Telecommunications Sector

\section{INTRODUCCIÓN}

\subsection{Situación actual del problema}

En 2009 el Gobierno español presentó su Anteproyecto de Ley de Financiación de la Corporación RTVE. Los sindicatos de la radiotelevisión pública llamaron inmediatamente la atención sobre el hecho de que el proyecto jurídico se había ido negociando con una evidente falta de trasparencia y sin la participación de los sectores concernidos: la SEPI, los sectores empresariales de las telecomunicaciones, las organizaciones de anunciantes, las demás fuerzas políticas. Tampoco fueron consultados el Consejo de Administración y el equipo directivo de la corporación. ${ }^{2}$

En resumen, se eligió el modelo adoptado por Francia con algunas diferencias: la ley francesa apostaba por la supresión paulatina de la publicidad en las cadenas públicas de televisión. La ausencia de anuncios en France Télévisions (France 2, France 3, France 4, France 5 y France Ô) es un hecho en Francia desde el 5 de enero de 2009 en la franja horaria que va desde las 20.00 hasta las 06.00 horas. Según las previsiones, la desaparición total de la publicidad se producirá el 30 de noviembre de 2011, coincidiendo con el "apagón analógico".

La nueva ley francesa crea un nuevo impuesto que grava con un $0,9 \%$ la facturación de los proveedores de telefonía e Internet. La tasa ha generado malestar en las empresas de telecomunicaciones, puesto que es superior al 0,5\% que había solicitado la comisión encargada de estudiar la supresión de la publicidad. Además, se aplica una tasa del $3 \%$ sobre la cifra de negocios por publicidad de las cadenas privadas cuyos ingresos superan los 11 millones de euros. A cambio, las televisiones privadas tienen derecho a un segundo corte publicitario cada 30 minutos en las emisiones de películas y series de ficción. A todo esto hay que sumar el canon que pagan todos los ciudadanos. ${ }^{3}$

En el caso español la supresión fue definitiva: la Corporación RTVE se financiará, vía

2 Ver: Comisiones Obreras ante el Proyecto de Ley de Financiación de RTVE, CCOO, 16 de junio de 2009.

${ }^{3}$ En 2009 el volumen de ingresos por publicidad de France Télévisions disminuye debido a la eliminación de la misma en la franja nocturna. Aplicado a la cifra neta de negocio, el Grupo France Télévisions pierde 3,9 puntos de cuota del mercado. En realidad, sus ingresos proceden de: recursos públicos (2. 412'4 millones de euros), publicidad y patrocinio ( $430^{\prime} 9$ millones de euros), otros (190'9 millones de euros). La disminución en el concepto de publicidad es de un 30,33 \% comparado con 2008 (618'5). La previsión para 2010 es de unos ingresos por publicidad de 343'6 millones de euros. (Informe anual del Grupo France Televisión. Disponible en

: http://www.francetelevisions.fr/downloads/FTV_rapport_financier_annuel_2009.pdf) 
Presupuestos Generales y a través de aportaciones obtenidas de la siguiente manera:

a) Los operadores de televisión comercial que operan a nivel estatal o en más de una Comunidad Autónoma aportarán anualmente un 3 por 100 de sus ingresos brutos ${ }^{4}$ La aportación para la televisión de pago está fijada en el 1,5 por 100.4 Los operadores de telecomunicaciones, también según el mismo criterio territorial, aportarán, por su parte, un 0,9 por 100 de sus ingresos brutos de explotación facturados en el año correspondiente, excluidos los obtenidos en el mercado de referencia al por mayor. ${ }^{5}$

b) La Corporación RTVE percibirá el 80 por 100 de lo recaudado por la tasa sobre reserva de dominio público radioeléctrico regulada en la Ley General de Telecomunicaciones.

c) La radiotelevisión pública contará también con los ingresos obtenidos por el ejercicio de sus actividades y por la comercialización de sus servicios y productos.

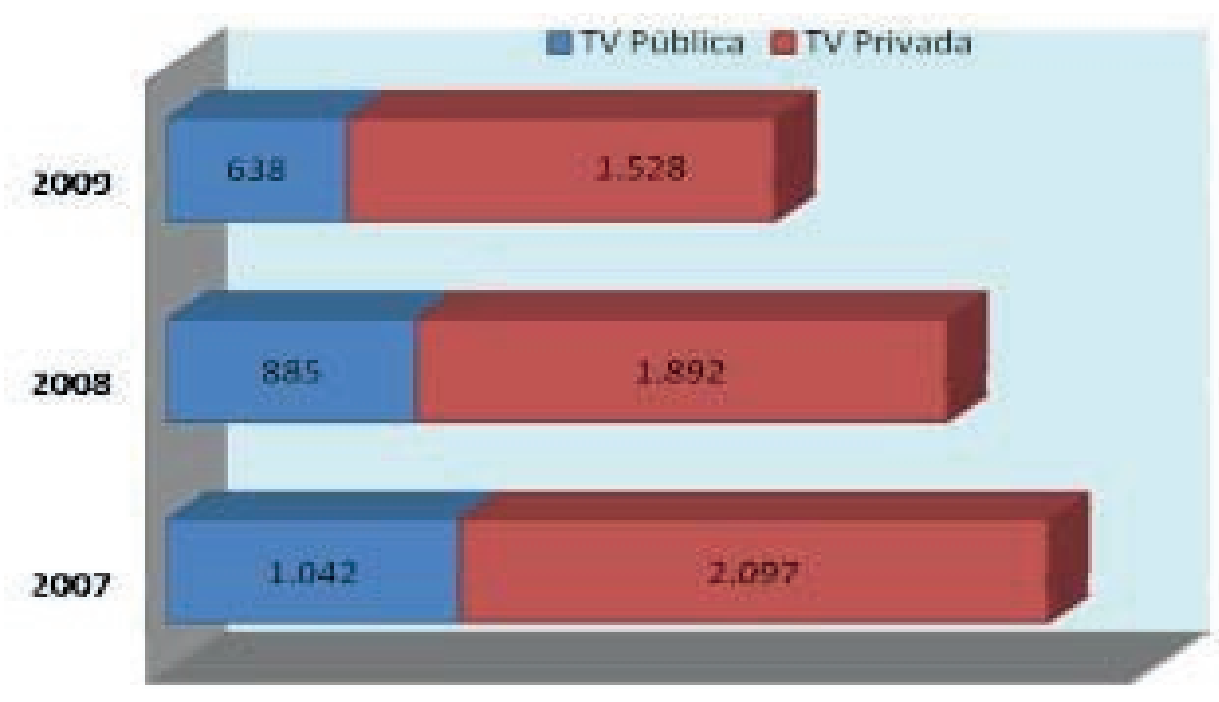

Figura 1. : Ingresos de la televisión pública y privada por publicidad: 2007- 2009

Fuente: CMT, Informe anual, 2009.

Con la entrada en vigor de los nuevos modelos de financiación, la Comisión Europea abría una investigación sobre el carácter de las ayudas estatales y sobre la legalidad de las nuevas tasas. Esto era necesario porque según la normativa europea "la financiación estatal de los organismos públicos de radiodifusión influye en el comercio entre Estados miembros. Esto es especialmente evidente en el caso de la adquisición y

\footnotetext{
${ }^{4}$ Esta aportación no podrá superar el 20\% del total de ingresos previstos para cada año en la Corporación RTVE

${ }^{5}$ En el caso de los operadores de telecomunicaciones, esta aportación no podrá superar el $25 \%$ del total de ingresos previstos para cada año en la Corporación RTVE
} 
venta de los derechos de difusión de programas, que a menudo se produce en el ámbito internacional" 6

A pesar del aumento en la compensación de servicio público en 2009 a 723 millones de euros lo que supone casi el 50\% más que el año anterior, la Comisión no considera el hecho una alteración sustancial del sistema aprobado por la misma en 2005 (ídem, p. 9). No obstante recuerda que en los casos en los que "un gravamen específicamente destinado a financiar la ayuda resulte contrario a otras disposiciones del Tratado, la Comisión no puede declarar el régimen de ayudas del que forma parte el gravamen compatible con el mercado interior" (ídem, p. 13). Esto significa que el método por el que se financia una ayuda puede hacer que todo el régimen de ayudas sea incompatible con el mercado interior.

La Comisión solicitó en su momento a España que no asignara ninguna financiación a RTVE que proceda de los impuestos introducidos por la Ley de Financiación hasta que se haya adoptado una decisión final. A finales de septiembre (2010) se hizo evidente que la Comisión iba a declarar ilegal la tasa impuesta a los operadores de telecomunicaciones. Por lo visto, la cuestión pasará en manos del Tribunal de Justicia lo que significa que de momento el nuevo modelo de financiación sigue en el aire. Al mismo tiempo, el Tribunal Supremo en España rechaza paralizar la aportación de los operadores de telecomunicaciones y los mismos de momento tendrán que pagar antes de reclamar ${ }^{7}$

En su comunicación de 30 de septiembre de 2010 la Comisión Europea declaró su decisión de exigir a Francia y España que supriman las tasas específicas sobre el volumen de negocios de los operadores de telecomunicaciones ${ }^{8}$

La Comisión cree que las tasas impuestas por Francia y España al efecto de compensar la pérdida de ingresos en concepto de publicidad de pago en los canales de televisión públicos son incompatibles con las normas de la UE en materia de telecomunicaciones. De conformidad con esas normas (en especial, el artículo 12 de la Directiva relativa a la autorización de redes y servicios de comunicaciones electrónicas (2002/20/CE), se pueden imponer tasas a los operadores de telecomunicaciones únicamente para sufragar determinados costes administrativos y

\footnotetext{
${ }^{6}$ Ayuda de Estado C 38/2009 (ex NN 58/2009) - España Nuevo sistema de financiación basado en los impuestos para los organismos de radiodifusión pública en España. C(2009)9313 final. Disponible en: http://ec.europa.eu/competition/state_aid/register/ii/doc/C-38-2009-WLAL-es-02.12.2009.pdf

7 La Comisión del Mercado de las Telecomunicaciones (CMT) aprobó en 2011 la suspensión de la ejecución de la liquidación complementaria de 709.821 euros que le impuso a Telefónica por la aportación de la tasa de financiación de RTVE correspondiente a 2009, alegando que ha presentado un recurso económico-administrativo

${ }^{8}$ Comisión Europea, IP/10/1211. La Comisión exige a Francia y España que acaben con los «impuestos sobre las telecomunicaciones. 30/09/2010, Bruselas. Disponible en: http:/ / europa.eu/rapid/pressReleasesAction.do?reference=IP/10/1211\&format=HTML\&aged=1\&lan guage $=$ ES\&guiLanguage $=\mathrm{fr}$
} 
reglamentarios (principalmente las autorizaciones y las funciones de reglamentación) y las tasas deben ser objetivas, transparentes y proporcionadas. Por otra parte, las partes interesadas también deben ser consultadas adecuadamente sobre cualquier modificación de las tasas cobradas a los operadores de telecomunicaciones (ibídem ${ }^{9}$.

En este artículo, tomando como base los antecedentes arriba mencionados, haremos un análisis de la situación actual de la Corporación RTVE. Para la elaboración del estudio, se ha recurrido a fuentes originales: Diario de Sesiones del Congreso de los Diputados, informes de la Comisión del Mercado de Telecomunicaciones, informes anuales de los medios de comunicación en España. Teniendo en cuenta que el objeto de investigación es la situación de la radiotelevisión pública en 2010, año para el que todavía no disponemos de unas cifras definitivas, ha sido necesario contrastar los datos con fuentes secundarias como publicaciones en la prensa y entrevistas con el Presidente de la Corporación y altos directivos difundidas por los medios de comunicación.

\section{METODOLOGÍA}

En la presente investigación se ha empleado el método histórico-lógico para realizar una caracterización del comportamiento en los últimos años de la situación económica- financiera de la radiotelevisión pública en España buscando al mismo tiempo ejemplos de gestión de países como Francia, Reino Unido y Alemania

Se realiza también un análisis cuantitativo para exponer la situación económica financiera de la corporación RTVE. Se empleó además el análisis documental para contextualizar la situación de esta corporación, así como para la discusión de los resultados.

\section{ANÁLISIS Y DISCUSIÓN}

\subsection{Situación económico-financiera de la corporación RTV}

Según los datos expuestos por el Presidente de la radiotelevisión pública ante la Comisión Mixta de Control Parlamentario de la Corporación RTVE, hasta octubre (2010) cuando finalizan los plazos para el ingreso de las aportaciones por parte de los operadores audiovisuales y de telecomunicaciones, el importe recaudado asciende al $81 \%$ del total estimado. En esta situación existe el peligro de cerrar el año con un posible déficit de 66 millones de euros:

\footnotetext{
${ }^{9}$ La tasa francesa sobre los operadores de telecomunicaciones se impuso en marzo de 2009 después de la decisión del Gobierno francés de poner fin a la publicidad de pago en los canales públicos de televisión. Se calcula que los ingresos anuales de la nueva tasa, que se abona al Tesoro francés, ascienden aproximadamente a 400 millones de euros. En el caso español, la tasa entra en vigor en septiembre de 2009 y se estima que genere unos ingresos de aproximadamente 230 millones de euros en 2010
} 
El 6 de octubre finalizó el plazo para que los operadores audiovisuales presentasen las autoliquidaciones, y para los dos primeros pagos a cuenta de 2010, así como para la presentación por parte de los operadores de telecomunicaciones del presente año. El importe estimado de ingresos a esa fecha era de 182,4 millones, el estimado. La recaudación efectiva que nos ha comunicado la Comisión del Mercado de Telecomunicaciones es 148,6 millones, con una diferencia negativa de 33,8 millones de euros. Asimismo en octubre de 2010 todos los operadores tenían que efectuar el tercer pago a cuenta de 2010, cuyo importe estimado era 78,7 millones, pero la recaudación efectiva notificada por la Comisión del Mercado de Telecomunicaciones también ha sido menor, 64,1 millones de euros, es decir, 14,6 millones de euros menos de lo esperado ${ }^{10}$

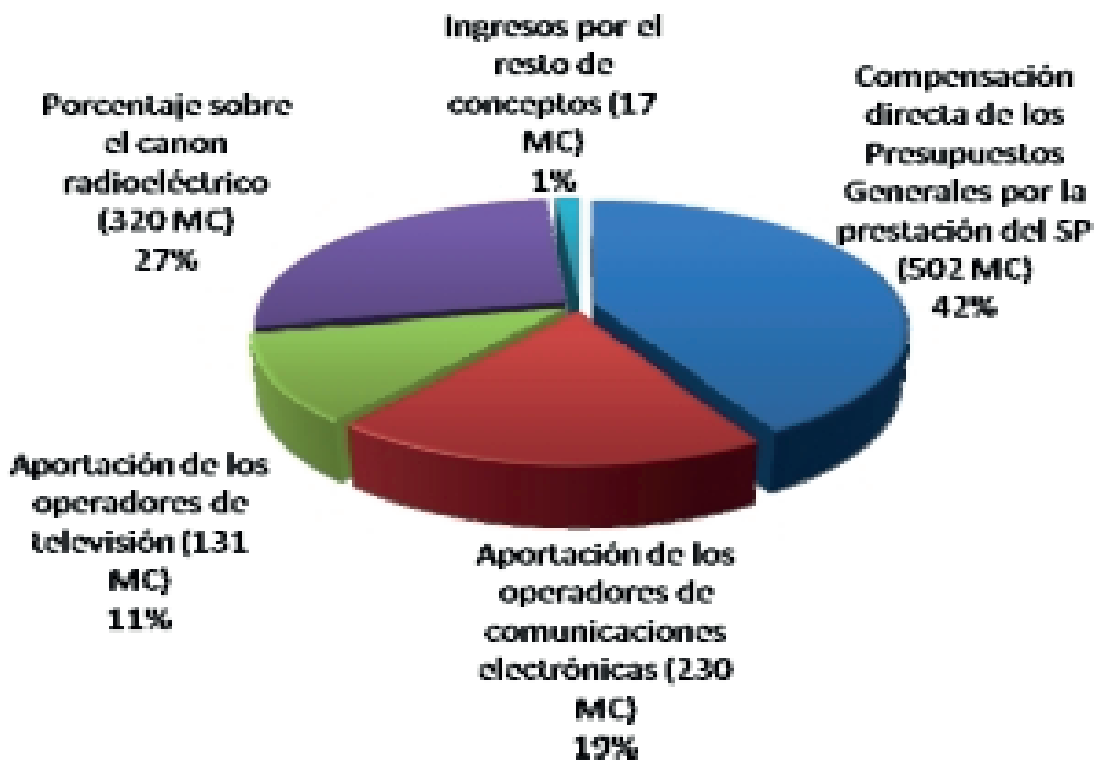

Figura 2. : Ingresos RTVE, 2010 (previsión) ${ }^{11}$

Fuente: Anteproyecto de ley de financiación de la corporación RTVE (2009).

Al mismo tiempo la corporación registra 25,7 millones (aprox. un 2\% sobre el presupuesto total) por venta de programas, venta de canales, material de archivo, vídeo doméstico, venta de doblajes y otros servicios, prestación de servicios de asistencia técnica, arrendamiento de inmuebles, propiedad intelectual, etc ${ }^{12}$

\footnotetext{
${ }^{10}$ Diario de Sesiones del Congreso de los Diputados: $\mathrm{n}^{\mathrm{o}} 161,21 / 12 / 2010, \mathrm{p} .4$

${ }^{11}$ Respecto a la tasa radioeléctrica encontramos la siguiente explicación del Presidente de la Corporación ante la Comisión de Presupuestos del Congreso de los Diputados: (Diario de Sesiones, 06/09/2010, no 618, p. 55)

12 Debemos subrayar que el presidente, A. Oliart, se ha pronunciado varias veces a favor de "la modificación de la Ley de Financiación según la cual todos los ingresos por la venta de derechos o contenidos son ingresadas directamente a las arcas públicas y no en las cuentas propias". En realidad, el art. 7 de la Ley 8/2009 de financiación de la Corporación de la Radio y Televisión Española establece lo siguiente: "Los ingresos derivados de lo establecido en los dos párrafos anteriores (se refiere a los ingresos derivados de la propia actividad, nota mía) se minorarán de las compensaciones por el
} 
Respecto al cien por cien del dominio público radio-eléctrico, los 330 millones que figuraban en el presupuesto de 1.200 millones no los pusimos nosotros porque sí, sino porque nos dieron esa cifra. Luego resultó que o había habido un error en la cifra o la recaudación fue menor durante este año de 2010 y en junio resultaba que era 225.5 y que eso nos producía un déficit del 78,9 por ciento. Si recuerda, era esa cifra 78,9, y la manera de aminorar el impacto de una cifra tan considerable en la baja de nuestros ingresos fue modificar el tipo y pasar del 80 al cien por cien, cosa con la que gratamente nos encontramos por un real decreto que así lo estableció. El Gobierno podría explicarle a usted por qué decidió ese camino; camino que a nosotros nos evitó una pérdida que hubiera sido difícilmente asumible en lo que quedaba de año 2010.

Según el anteproyecto de presupuestos de la corporación para 2011 aprobado por el Consejo de Administración, RTVE recibirá este año unos 550 millones de euros de aportación del Estado. La radiotelevisión pública prevé disponer de los 1.200 millones de euros que le permite como máximo la ley, gracias a las aportaciones de los "telecos" (unos 250 millones) y de las cadenas privadas (100), así como de la tasa radioeléctrica (unos 314 millones). Los 1.200 millones se completarán con los 27 millones que se calcula obtener de la comercialización de productos ${ }^{13}$

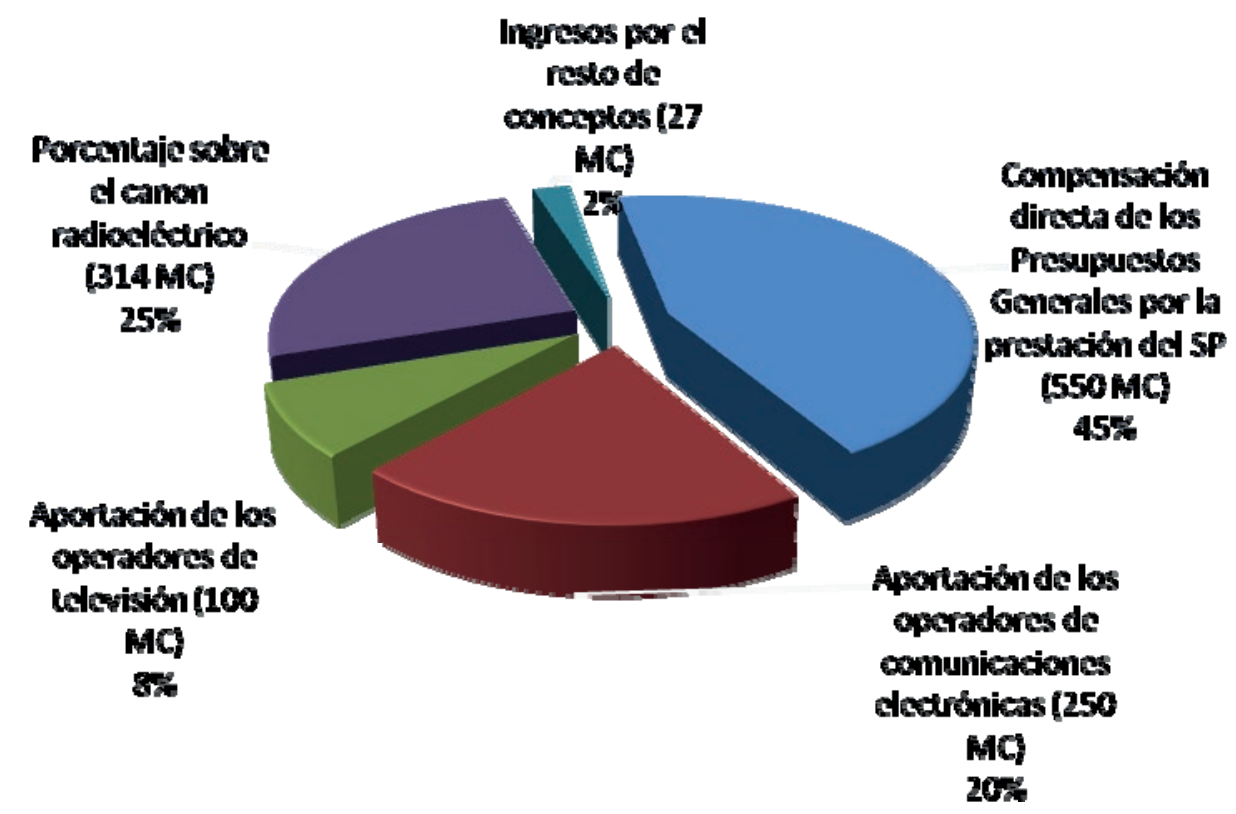

Figura 3. : Ingresos RTVE, 2011 (previsión)

Fuente: Elaboración propia a partir de las fuentes

Al mismo tiempo, la SEPI (Sociedad Estatal de Participaciones Industriales) destinará en 2011 un presupuesto de 107 millones a financiar los gastos que genera el antiguo Ente Público de Radiotelevisión dentro del plan de financiación de la deuda que puso

cumplimiento de las obligaciones de servicio público consignadas en los Presupuestos Generales del Estado".

13 Fuente: Servimedia, Periodista Digital: "RTVE recibirá 35 millones menos del Estado en 2011"

(22/09/2010). Disponible en: http://www.periodistadigital.com/periodismo/tv/2010/09/22/rtve-

recibira-35-millones-menos- del-estado-en-2011.shtml 
en marcha el Gobierno en 2007.14 Debemos recordar que simultáneamente con la elaboración del proyecto de una nueva ley en 2004- 2005, desde el Ministerio de Economía y de la SEPI se diseñó un plan financiero para asumir la deuda acumulada de RTVE y aumentar la subvención estatal hasta cubrir los costes del servicio público:

Para el año 2011 los gastos del ERE que figuran del ente público serán 178 millones. Hay además otro gasto de estructura que será de 2,5 millones. Por otra parte, el ente público recibe una financiación de 82 millones directamente del Ministerio de Economía. Hay una pérdida de 98.543 .000 euros que se financian por la compra de las acciones que SEPI realiza al ente público con la dotación que le da el presupuesto del Ministerio de Economía, a la que se refería anteriormente. Al final de estas operaciones, que empezamos en el año 2007, SEPI ha ido adquiriendo, porque es la fórmula de financiar la carga de la deuda histórica de la televisión, las acciones que recibió el ente cuando le dio todo su activo a la corporación. Al final del año 2010 la participación de SEPI a través de este procedimiento será de 50,06 por ciento y en el año 2011 del 52,68 por ciento (el Presidente de la SEPI ante la Comisión de Presupuestos del Congreso de los Diputados, ibídem).

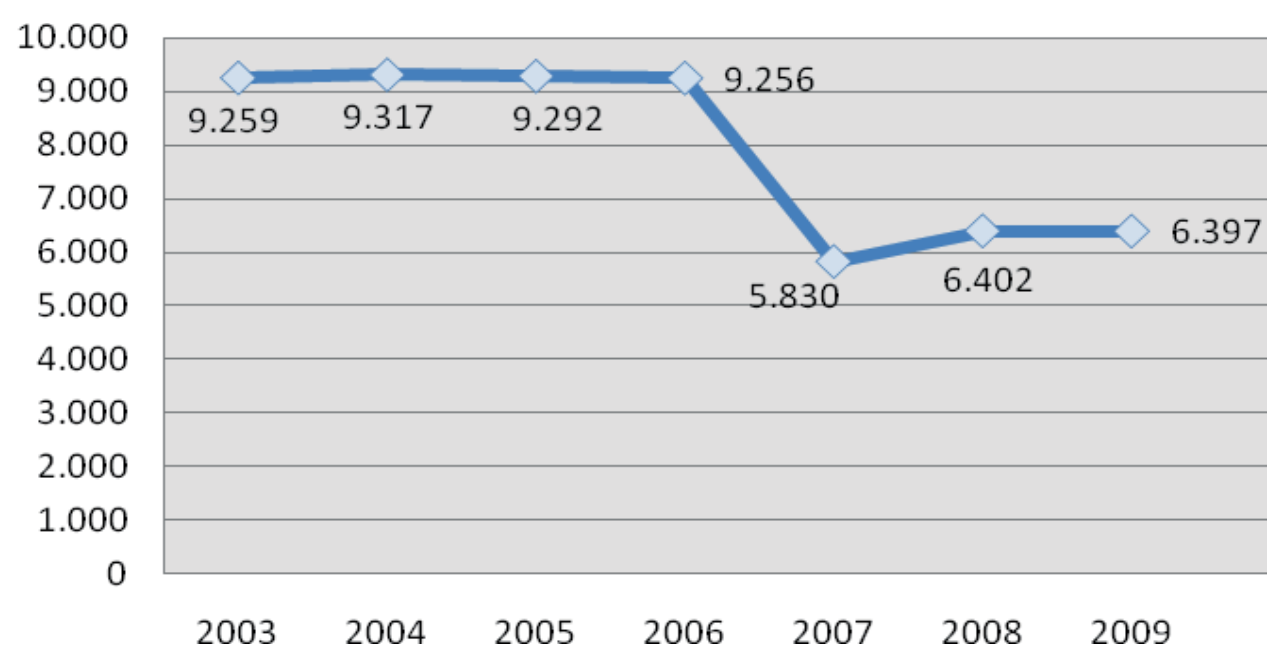

Figura 4. Reducción de las fuerzas de trabajo en RTVE:

Fuente: Informes anuales de la CMT; informes anuales de RTVE

En la Ley 42/2006, de 28 de diciembre, de Presupuestos Generales del Estado para el año 2007, se decide que el Estado asumiera por un importe máximo de 3.180.910,91 millones de euros el nominal de las operaciones de endeudamiento del Ente público RTVE. Se prevé también que durante los ejercicios de 2008 a 2010 se asumiera el importe nominal restante de la deuda acumulada a 31 de diciembre de 2006.

En resumen: en el año 2007 se asumen por el Tesoro Público 3.181 millones de euros que corresponden a la deuda histórica que a finales de 2007 va a alcanzar la cifra de

${ }^{14}$ Diario de Sesiones, 06/09/2010, nº 618, p. 43 
7.811 millones de euros. El resto, hasta llegar a la cifra de 7.811, será asumido por el Tesoro en los años 2008, 2009 y 2010 hasta completar la asunción de la deuda

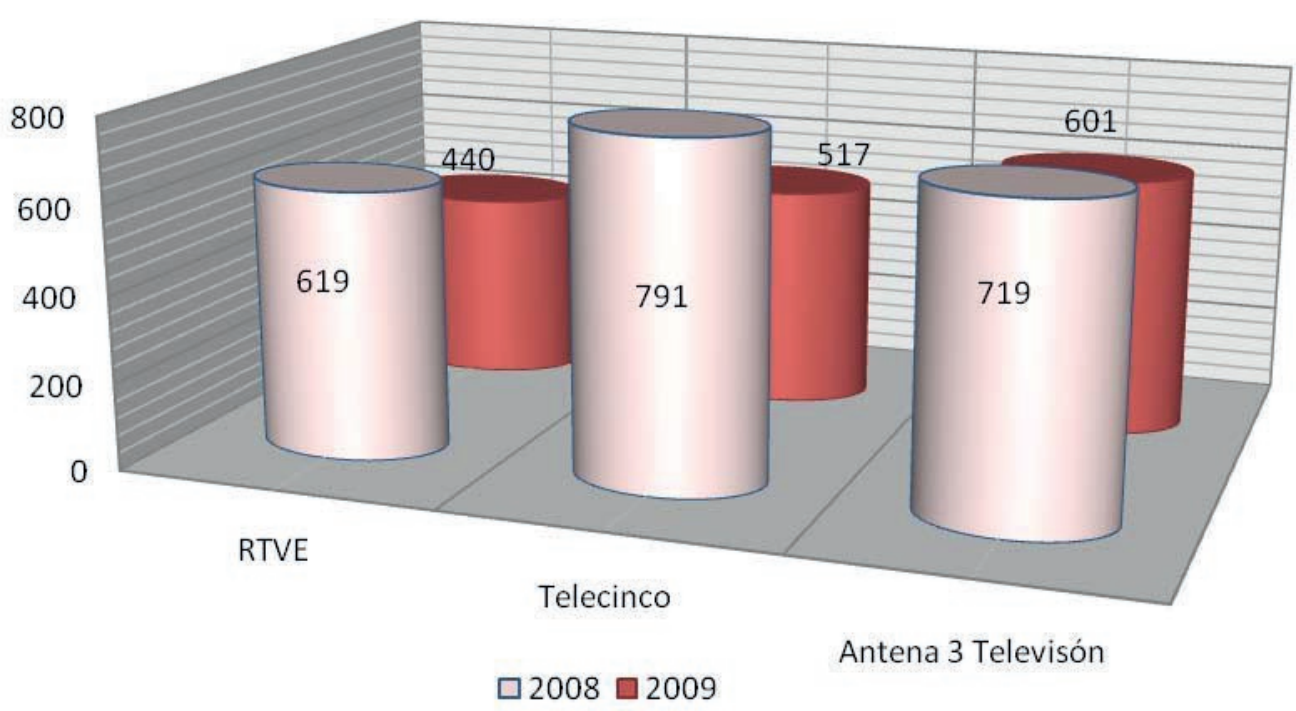

Figura 5. Endeudamiento de RTVE $(2008,2009)$ en comparación con las dos cadenas generalistas privadas de mayor audiencia (en ME):

Fuente: Informe anual de la Comisión del Mercado de las Telecomunicaciones (http://www.cmt.es/cmt_ptl_ext/SelectOption.do?nav=publi_anuales)

Cambiando de asunto, hay que destacar que el mandato-marco de RTVE fijó los siguientes objetivos de producción interna y externa en la producción de programas y contenidos (art.2): a) El 100\% de producción interna en programas informativos, programas de carácter institucional y contenidos de autopromoción. b) El 60\% de producción interna de la oferta generalista del conjunto de la programación con contenidos de actualidad, infantil y juvenil, educativo, medioambiental, religioso o de protección al consumidor. c) El 55\% de producción interna de la oferta generalista del conjunto de los programas de género divulgativo, deportivo, ficción, series, magazines, concursos, humor, entretenimiento gran formato, talk show, ficción dibujos animados, docu-show, concurso gran formato, musical, docu-serie, reality show, televenta y ficción teatro.

Durante su aparición ante la Comisión Mixta de Control Parlamentario en el mes de octubre, el Presidente de la corporación explicó que en el año 2008 los porcentajes de producción propia fueron de 72,81\%; la mixta del 1,61\% y la ajena del 25,59\%; en 2009 se situaron en el 73,43\% para la propia, 6,59\% para la mixta y 19,98\% para la ajena. Durante el primer cuatrimestre, la cifra de la producción propia es 69,38\%, la mixta $13,90 \%$ y la ajena $16,72 \% .{ }^{15}$

Analizando los datos en términos de producción interna y externa como nuevos criterios de gestión de recursos, arrojan los siguientes resultados: en 2008, un 70,44\% la

15 Diario de Sesiones del Congreso de los Diputados, $n^{\text {o }} 149,27 / 10 / 2010$, p. 2 
interna y un 29,56\% la externa. En 2009, la interna supuso el $63,81 \%$ y la externa el $36,19 \%$. Finalmente, en el primer cuatrimestre de 2010 los porcentajes se sitúan en el $62,37 \%$ para la producción interna y en el 37,63\% la externa. Este aumento de la producción externa se ha convertido en uno de los asuntos más polémicos para la corporación.

\subsection{El canon como vía de rescate}

Es necesario ver la cuestión del canon aparte por la polémica causada por las voces a favor de su introducción como fuente de financiación para la radiotelevisión pública. Con este fin, analizaremos como ha sido resuelto el problema en países como Alemania y Reino Unido.

En 2007, la Comisión Europea dictó que la definición de las obligaciones de servicio público en el Convenio Nacional de Radiodifusión en Alemania es muy general con la pretensión de que sean luego los Estados federales los que introduzcan los detalles (Schulz, 2008). El Gobierno alemán respondió que el pago del canon por parte de los ciudadanos no supone una ayuda estatal porque el mismo va destinado al cumplimiento de ciertos compromisos a favor de la sociedad. Esa diferencia abrió paso a una discusión que terminó con el llamado compromiso sobre los subsidios (Beihilfekompromiss). Alemania se comprometió a introducir los mecanismos para una mejor definición de las obligaciones de servicio público.

En todo esto es determinante que sean los Estados federales los que tienen la obligación de fijar la cantidad del canon para el propio territorio respetando la legislación común (el Convenio Nacional de la Radiodifusión y el Convenio Nacional para la ZDF) que establece los principales mecanismos para llevar a cabo el proceso. Las leyes federales (Rundfunkgesetze der Ländern) nunca deben crear una situación conflictiva de cara a las normas a nivel nacional.

El proceso de cálculo del canon viene definido en el Convenio Nacional sobre el Canon Audiovisual (Rundfunkgebührenstaatsvertrag). Una comisión independiente de 16 miembros (Kommission zur Überprüfung und Ermittlung des Finanzbedarfs der Rundfunkanstalten, KEF) emite una propuesta sobre la cantidad del canon para cada estado federal basada en la descripción de la situación financiera de cada una de las cadenas públicas de radiodifusión y las necesidades expuestas por las mismas. De esta manera se calcula la cantidad del canon para periodos de cuatro años. 


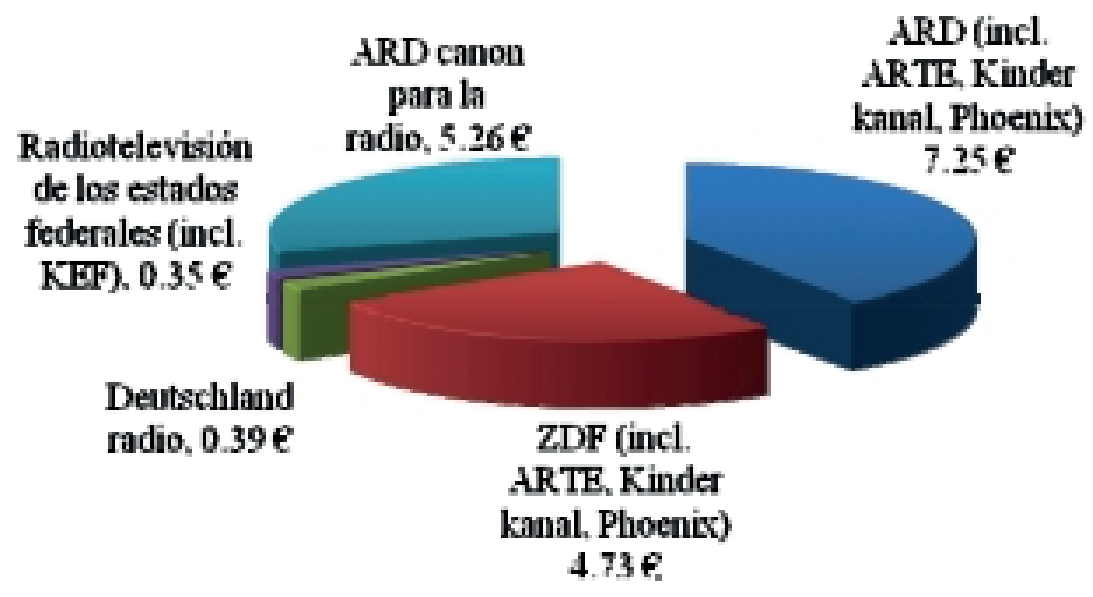

Figura 6. Reparto del canon mensual en Alemania (a partir de 2009)

Fuente: V. Berger, 2008

El canon en Reino Unido constituye la principal fuente de financiación para la radiotelevisión pública aunque no la única. Como podemos observar en el siguiente gráfico, la BBC cuenta también con otras fuentes de ingresos

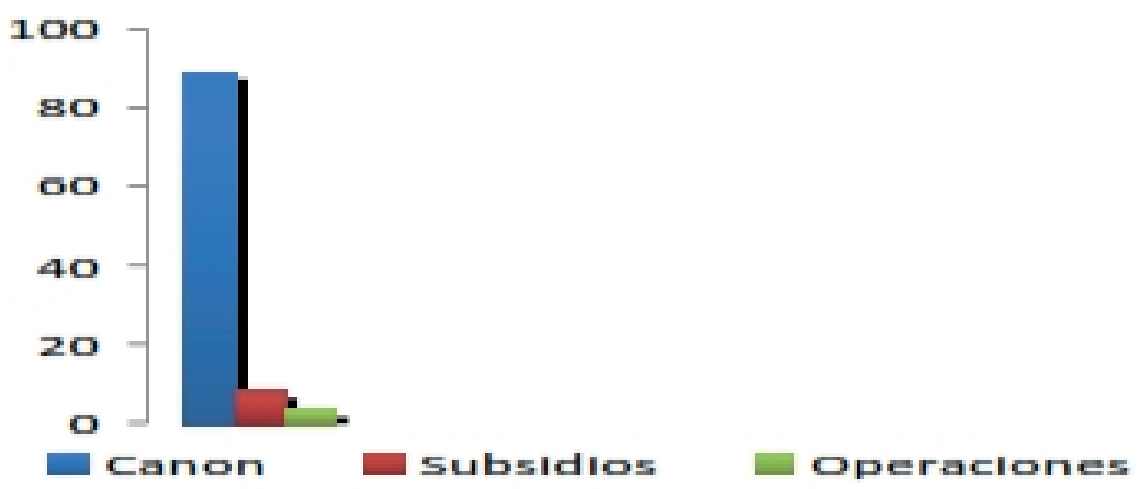

Figura 7. Ingresos (\%) de la BBC (2009/2010) ${ }^{16}$

Fuente: BBC, cuentas anuales 2009/2010

El canon anual para el año fiscal 2009/2010 fue de 139.50 libras esterlinas. A partir de 1 de abril de 2009 la cantidad subió a 142.50 libras esterlinas

\footnotetext{
${ }^{16}$ En el apartado de operaciones comerciales incluimos a la BBC Worldwide (la iniciativa privada de la $B B C$ que se encarga de una amplia gama de actividades desde la inversión hasta la distribución y venta de programas), BBC Studios and Post Production (destinada a colaborar con operadores privados del sector en la elaboración de productos) y a la BBC World News (canal financiado por una tasa de suscripción y por ingresos de publicidad). Los subsidios van destinados a la BBC World Service
} 


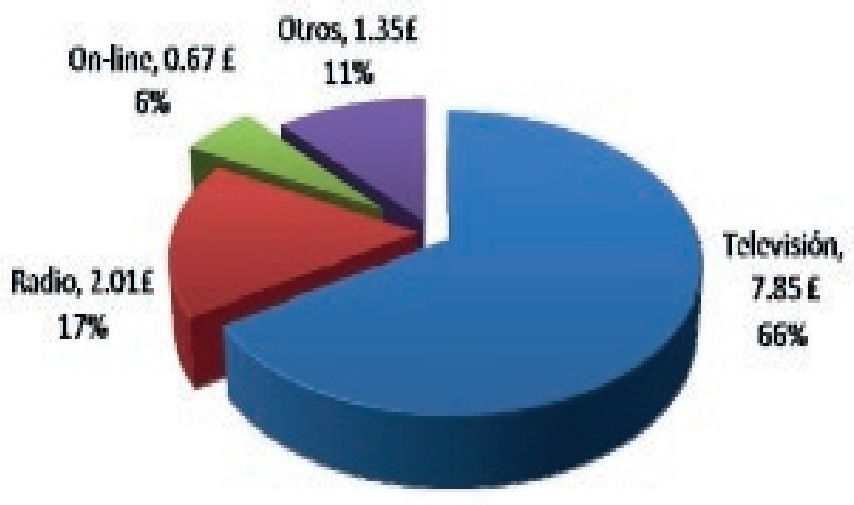

Figura 8. Ingresos (\%) de la BBC (2009/2010

Fuente: BBC Annual Report 2009/2010

Hablando del canon, consideramos necesario ofrecer algunos datos orientativos con el fin de percibir una idea general del cargo económico que supondría para las familias la posible introducción de este mecanismo de financiación en España. En primer lugar, debemos señalar que existen distintos escenarios que dependen de diversas variables. Si se aprueba, por ejemplo, un modelo de canon por vivienda con televisión (con el canon como única vía de financiación de un presupuesto total de 1.200 millones de euros) esto generaría una tasa de 79 euros por vivienda al año $\left(6,58\right.$ euros al mes). ${ }^{17}$

Por otra parte, el canon podría sustituir únicamente la compensación directa de los Presupuesto Generales y la aportación de los operadores de telecomunicaciones (en el caso en el que el Tribunal de Justicia confirmara la ilegalidad de la tasa impuesta por la Ley 8/2009) conservando el porcentaje sobre el canon radioeléctrico y la aportación de los operadores de televisión privada. Entonces, el canon vendría a compensar alrededor de 800 millones de euros de un presupuesto total de 1.200 millones de euros. En este último caso, a cada vivienda con televisión correspondería pagar 52 euros al año (4,37 euros al mes). El reparto de las fuentes de financiación sería el siguiente:

\footnotetext{
${ }^{17}$ Según los últimos datos del Instituto Nacional de Estadística, en España hay un total de 15.248.658 viviendas que disponen de televisión. Ver: http://www.ine.es/
} 


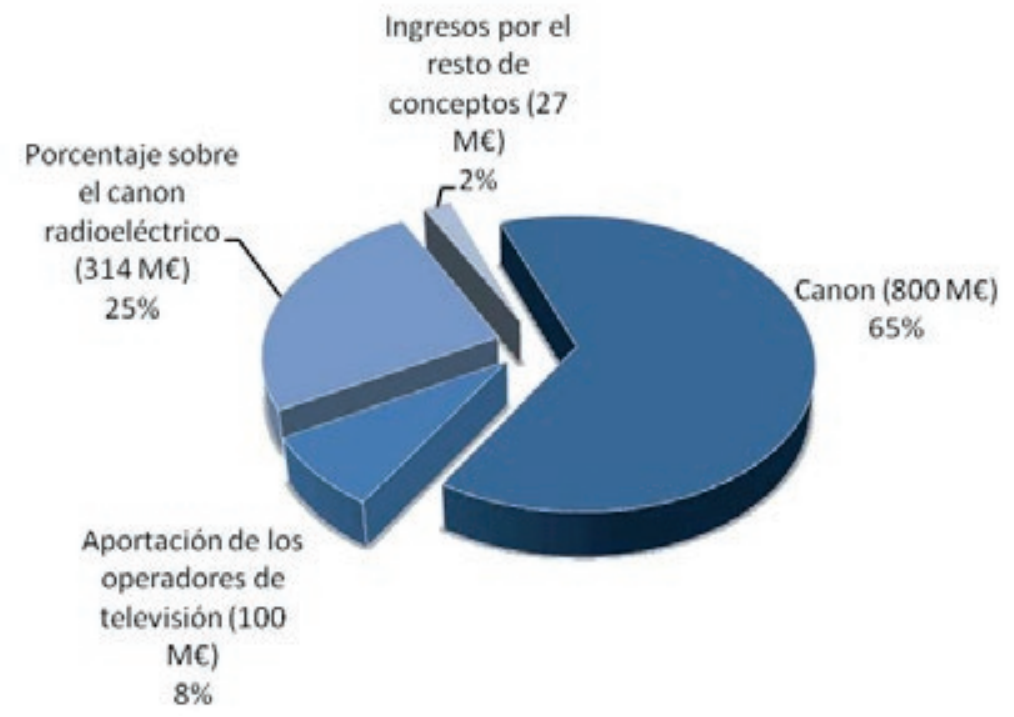

Figura 9. El canon como vía de financiación de la Corporación RTVE (previsión basada en datos financieros de 2010-2011)

Finalmente, debemos señalar que en estas estimaciones hemos dejado aparte la radiotelevisión pública autonómica. Según datos difundidos por la prensa a finales de 2010, las cadenas autonómicas tienen un coste que se sitúa entre los 54- 253 euros por familia dependiendo de la Comunidad Autónoma ${ }^{18}$

Debemos señalar que la televisión pública sin publicidad goza de buena aceptación tomando como punto de valoración los índices de audiencia

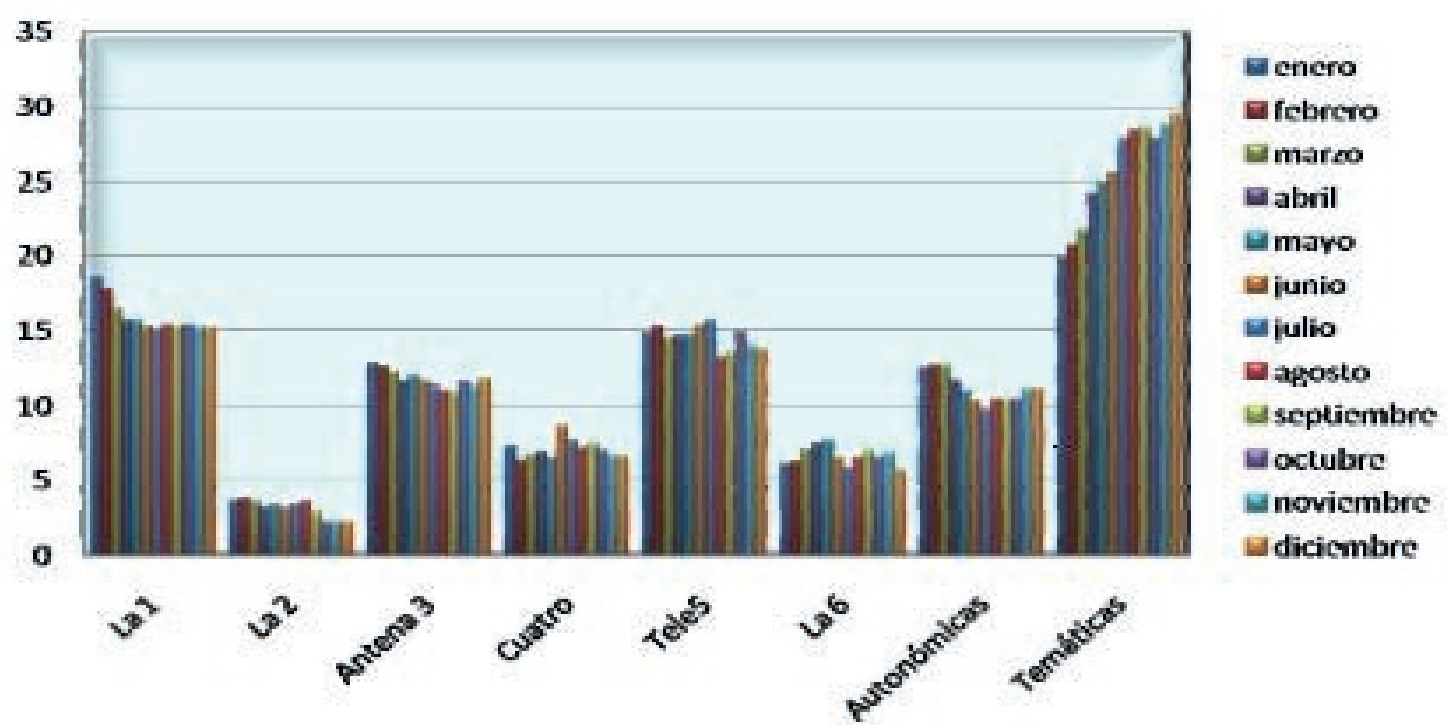

Figura 10. Share mensual de las cadenas de televisión en 2010

Fuente: elaboración propia a partir de las fuentes (FormulaTV: http://www.formulatv.com/)

\footnotetext{
${ }^{18}$ Fuente Europapress, 27/09/2010. Disponible en: http://www.europapress.es/aragon/noticiaconsejero-presidencia-dice-television-autonomica-cuesta-75-euros-anuales-familia-20100927132851.html
} 
Como corolario de todo lo dicho respecto de la situación actual de RTVE no podemos dejar de citar un artículo de una de las personas que formó parte del comité para la reforma del ente, E. Bustamante, y que fue publicado por Le Monde Diplomatique en febrero de $2010^{19}$

se prohibía que el presupuesto de gastos de RTVE superara los 1.200 millones de euros en 2010-2011, ni aumentará más de un 1\% anual en el siguiente trienio. Y todo ello a semanas vista del apagón analógico, tras el que RTVE deberá alimentar tres programas más (hasta 8), y con el añadido de tener que llenar con contenidos propios las horas de programación dejadas vacías por la publicidad

Las palabras de E. Bustamante encuentran confirmación en una de las respuestas del Presidente de RTVE, A. Oliart, ante la Comisión Mixta de Control Parlamentario de la Corporación de donde percibimos claramente las dificultades a las que se enfrenta la institución para hacer planes a largo plazo. La explicación es acerca de los contenidos previstos para el segundo múltiplex:

Hay tres posibilidades y una de ellas creo que será la que al final se va a elegir. Es el mantenimiento de la estructura actual, quedando abierta la posibilidad de incorporar en el segundo múltiplex un canal en definición estándar, emisión del canal Teledeporte en alta definición y mantenimiento de la estructura actual, pero fortaleciendo la señal en alta definición del propio canal HD y del Teledeporte. Yo creo que nos dirigiremos hacia esta solución mixta, es decir, básicamente deportes, pero también todas las cosas importantes que puedan darse en un momento dado en TV1 y nos interese darlas en alta definición, incluso alguna película que consideremos prime time extraordinaria ${ }^{20}$

\section{CONCLUSIONES}

Finalmente, debemos recordar que ante el modelo de financiación de la radiotelevisión pública en España hay muchas incógnitas. Probablemente, el Tribunal de Justicia confirmará la decisión de la Comisión Europea de declarar la tasa a los operadores de telecomunicaciones ilegal lo que supondría la necesidad de una nueva vía de financiación para compensar la eliminación de los 250 millones de euros, el $20 \%$, del esquema. A día de hoy es muy difícil hacer una previsión acerca de un futuro escenario ya que se han oído distintos planteamientos: unas a favor de devolver la publicidad a RTVE, otras proponiendo que sea el Estado quien deba compensar la diferencia y por último- la introducción de un canon a pagar por los ciudadanos como la vía más viable de financiación del servicio público audiovisual

\footnotetext{
${ }^{19}$ Le Monde Diplomatique (edición en español), n ${ }^{\circ}$ 172, febrero de 2010, "La contrarreforma audiovisual socialista"

${ }^{20}$ Diario de Sesiones, № 149, 27/10/ 2010, p. 16
} 


\section{REFERENCIAS}

Bardoel J.\& d'Haenens L. S. (2009). Reinventing Public Service Broadcasting in Europe: Prospects, Promises, and Problems, ponencia presentada en The Annual Meeting of the International Communication Association, Dresden.

Barnett S. (2007). Can the Public Service Broadcasting Survive? Renewal and Compromise in the BBC Charter. En G Lowe. \& J. Bardoel (Eds.), From Public Service Broadcasting to Public Service Media (pp. 87- 104). Göteborg: Nordicom Göteborg University

Berger V.(2008). Der deutsche Fernsehmarkt. Raderborn: Wilhelm Fink.

Bustamante, E. (2008).Public Service in the Digital Age: Opportunities and Threats in a Diverse Europe. En I Fernández. \& M. Moragas (Eds.), Communication and Cultural Policies in Europe (pp. 185- 216). Barcelona: Generalitat de Catalunya

Bustamante, E. (2010). La contrarreforma audiovisual socialista. Le Monde Diplomatique (esp.), 172, 23-40

Comisión Europea, IP/10/1211 (2010, 30 de Septiembre). La Comisión exige a Francia y España que acaben con los «impuestos sobre las telecomunicaciones. Bruselas. Consultado el 30 de Septiembre del 2010, Disponible en:

http:/ / europa.eu/rapid/pressReleasesAction.do?reference=IP/10/1211\&format $=H T$ $\underline{\text { ML\&aged=1\&language }=\text { ES\&guiLanguage }=\mathrm{fr}}$

Campos Freire, F. \& López Cepeda A. (2009). La reforma de la financiación de la televisión pública en Europa. En Madrid. VII Congreso Internacional ULEPICC Políticas de cultura y comunicación: creatividad, diversidad y bienestar en la Sociedad de la Información.

COM (2001) C 320/04: Comunicación de la Comisión sobre la aplicación de las normas en materia de ayudas estatales a los servicios públicos de radiodifusión.

Consideraciones sobre el Informe de la Comisión del Mercado de Telecomunicaciones al Anteproyecto de Ley de Financiación de la Corporación de Radio y Televisión Española, Ministerio de la Presidencia, 25 de mayo de 2009.

Diarios de Sesiones del Congreso de los Diputados. Consultado el 30 de Septiembre del 2010. Disponible en:

http://www.congreso.es/portal/page/portal/Congreso/Congreso/Publicaciones/D $\underline{\text { iaSe }} \underline{\mathrm{s}}$

Informe a la Vicepresidencia primera del Gobierno y Ministerio de la Presidencia sobre el Anteproyecto de Ley de financiación de la Corporación de Radio y Televisión Española (2009), Comisión del Mercado de Telecomunicaciones, Expediente RO 2009/747. Consultado el 21 de Mayo de 2009, Disponible en: 
http://www.cmt.es/es/resoluciones/18-2009/PRO_4758472/RE-2009-521-8-1.htm

Informe anual del Grupo France Télévisions. Consultado el 1 de Mayo de 2010, Disponible en:

http://www.francetelevisions.fr/downloads/FTV_rapport_financier_annuel_2009.pd $\underline{f}()$.

Ley 32/2003, de 3 de noviembre, General de Telecomunicaciones. Consultado el 1 de Abril de 2010, Disponible en:

http://www.boe.es/aeboe/consultas/bases_datos/doc.php?id=BOE-A-2003-20253.

Ley 17/2006, de 5 de junio, de la radio y la televisión de titularidad estatal. Consultado el 1 de Abril de 2010, Disponible en:

http://www.rtve.es/files/70-9847-FICHERO/Ley_radio_television_estatales.pdf

Ley 8/2009, de 28 de agosto, de financiación de la Corporación de Radio y Televisión

Española. Consultado el 31 de Agosto del 2009, Disponible en:

http://www.boe.es/boe/dias/2009/08/31/pdfs/BOE-A-2009-13988.pdf

Ley 7/2010, de 31 de marzo, General de la Comunicación Audiovisual. Consultado el 30 de Septiembre del 2010, Disponible en:

http://noticias.juridicas.com/base_datos/Admin/17-2010.html

Lowe G. \& Bardoel J. (2007).From Public Service Broadcasting to Public Service Media. Göteborg: Nordicom Göteborg University.

Manfredi Sánchez, J. L. (2008). La televisión pública en Europa. Madrid: Ediciones y Publicaciones Autor.

Medina, M. \& Ojer, T.(2009). Valoración del servicio público de televisión. Comparación entre la BBC y TVE. RLCS. Revista Latina de Comunicación Social, 64, 275299.

Schulz W. (2008): Der Programmauftrag als Prozess seiner Begründung. Vorschläge zu Verfahren und Organisation des »Drei-Stufen-Tests « zur Selbstkonkretisierung des Funktionsauftrags öffentlich-rechtlicher Rundfunkanstalten. Berlín, 2008. Kurzstudie im Auftrag der Friedrich- Ebert-Stiftung.

Semova D.(2010). Financing Public Media in Spain: New Strategies. International Journal on Media Management, (141), 3-12. 


\section{Dimitrina Jivkova Semova}

Doctora en Ciencias de la Información por la Universidad Complutense de Madrid. Licenciada en Periodismo por la Universidad de Sofía y Comunicación Audiovisual por la Universidad Complutense de Madrid; Diploma de Estudios Avanzados en Planteamientos Teóricos, Estructurales y Éticos de la Comunicación de Masas por la Universidad Complutense de Madrid. Becaria del Programa de Formación de Profesorado Universitario del Ministerio de Educación y Ciencia. Estancias de investigación en la Freie Universität de Berlín y en la Universidad de Harvard. Participación en diversos proyectos de investigación relacionados con el servicio público de radiotelevisión, usos de los medios de comunicación, necesidades de la audiencia. 